\title{
We Asked the Experts: Covid-19 Outbreak: Is There Still a Place for Scheduled Surgery? "Reflection from Pathophysiological Data"
}

\author{
Emmanuel Besnier $^{1,2} \cdot$ Jean-Jacques Tuech ${ }^{3,4} \cdot$ Lilian Schwarz ${ }^{3,4}$
}

Published online: 3 April 2020

(C) Société Internationale de Chirurgie 2020

\section{Introduction}

Covid-19 pandemic severely hits the world, and many countries need to adapt their health systems to handle a large influx of patients with pneumonia due to this new virus. This unique crisis is putting a strain on our hospitals, which have to respond to the influx of patients with Covid19. At the same time, physicians must provide care for patients suffering from other, and sometimes urgent, conditions. In this context, the management of patients requiring major oncological or cardiac surgery within adapted (often short) timeframes is a difficult preoccupation, especially since that some pathophysiological data on this virus leads us to believe that immunosuppressive changes due to surgery may increase susceptibility to the virus.

In this article, we present areas for reflection regarding the management of these patients during the Covid-19 pandemic situation, based on the little-known pathophysiological data on this virus.

Emmanuel Besnier

emmanuel.besnier@chu-rouen.fr

1 Normandie Univ, UNIROUEN, Inserm U1096, FHUREMOD-VHF, 76000 Rouen, France

2 Department of Anesthesiology and Critical Care, Hôpital Charles Nicolle, Rouen University Hospital, 1 Rue de Germont, 76031 Rouen Cedex, France

3 Department of Digestive Surgery, Rouen University Hospital, 1 Rue de Germont, 76031 Rouen Cedex, France

4 Department of Genomic and Personalized Medicine in Cancer and Neurological Disorders, Normandie Univ, UNIROUEN, UMR 1245 INSERM, Rouen University Hospital, 76000 Rouen, France
COVID-19 is a virus of the $\beta$-coronavirus family and is responsible for the third human epidemic of coronavirus zoonosis after SARS-Cov (2002) and MERS-Cov (2012). It is more precisely named Severe Acute Respiratory Syndrome Coronavirus 2 (SARS-Cov-2) because of its high sequence homology with SARS-Cov, sharing $80 \%$ genetic similarities [1] and equivalence in physiophathological mechanisms. All continents are currently affected, and the World Health Organization estimates that 179,111 cases are currently confirmed and 7426 deaths have occurred as of 17 March 2020. These figures are most probably underestimated since $80 \%$ of cases may be pauci-symptomatic and $34 \%$ asymptomatic [2]. Severe forms with respiratory failure can constitute up to $15 \%$ of cases and would be more frequent in patients with cardiovascular comorbidities, diabetes or advanced age [2], which can evolve into an Acute Respiratory Distress Syndrome (ARDS) in a rapid manner. Pathological analysis of a deceased patient revealed the presence of typical ARDS lesions with tissue edema, epithelial desquamation and hyaline membrane formations, associated with lymphocyte infiltration [3]. Due to the high similarity with SARS-Cov (angiotensin-converting enzyme 2 as receptor to penetrate into pulmonary epithelial, leukocytes or endothelial cells), we can assume that cellular damages are equivalent, with rapid viral replication within the pulmonary epithelial cells responsible for capillary leakage, cell apoptosis and a local pro-inflammatory process responsible for lymphocyte influx. In addition, Covid-19 patients present a particular immune profile with lymphopenia, mainly to the detriment of CD4+ and CD8+ T lymphocytes, but also B and NK lymphocytes [2,4] and hyperneutrophilia with an increase in the Neutrophil/Lymphocyte (N/L) ratio. At the same time, Covid-19 patients present an unbalanced cytokine profile with a predominantly proinflammatory response 


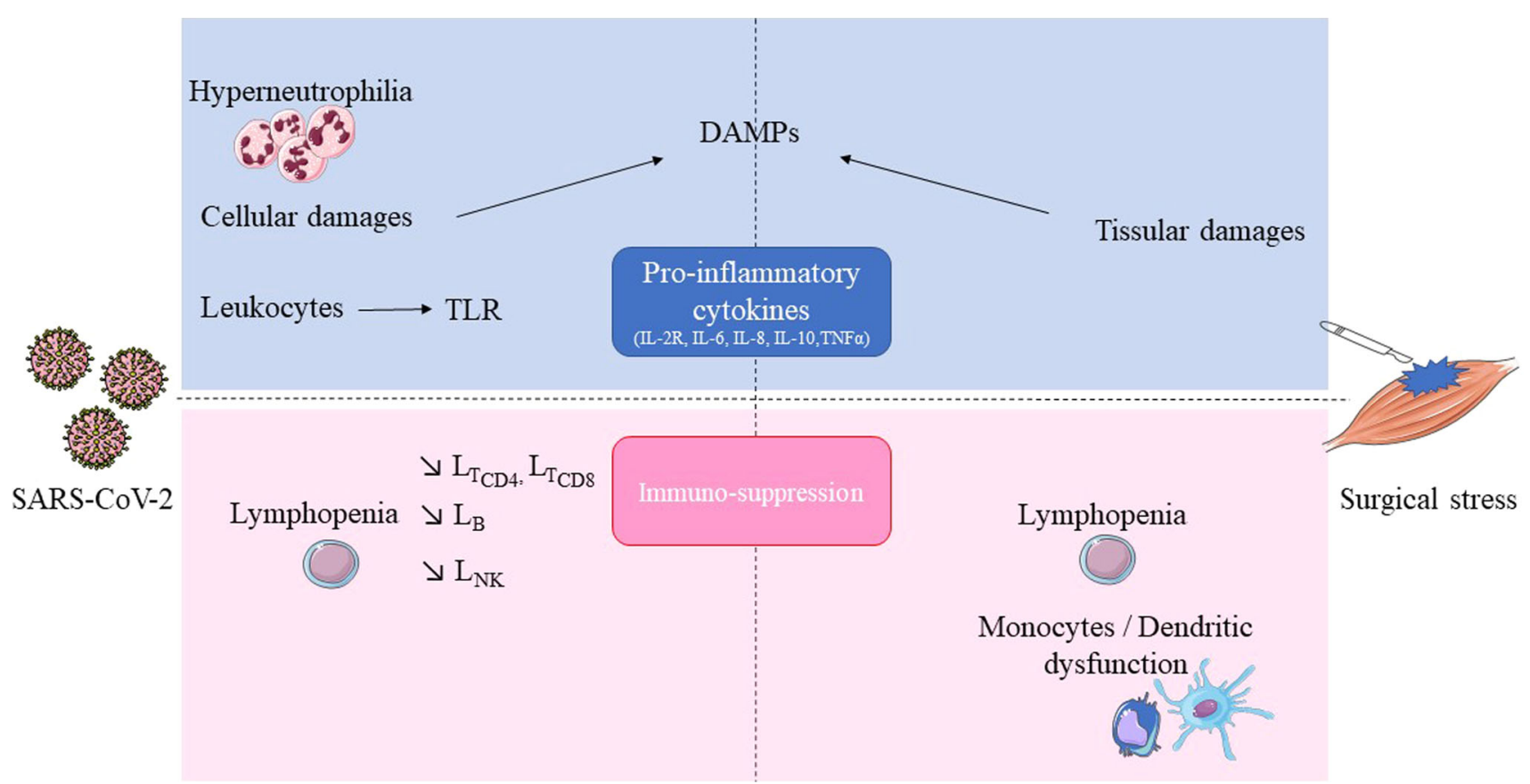

Fig. 1 Schematic representation of the main pathophysiological changes induced by both SARS-CoV-2 and surgery. DAMPs, damage associated molecular patterns; IL, interleukins; $\mathrm{L}_{\mathrm{T}}$, lymphocytes $\mathrm{T}$;

(Tool Like Receptor 3 and inflammasome activation; Damaged Associated Molecular Patterns and MitogenActivated Protein Kinases pathway). Qin et al. [4] demonstrated in a cohort of 452 Covid-19 patients an increase in levels of interleukins IL-2R, IL-6, IL-8, IL-10 and TNF $\alpha$. In particular, IL-6 levels were especially high in the most severe patients $(+90 \%$ versus non-severe patients). This point is crucial because IL-6 is known to be highly associated with the severity of critically ill patients, the onset of organ failure and myocardial dysfunction during sepsis [5].

Surgical stress is associated with a change in the immune profile. Patients undergoing major surgery, particularly cardiac, thoracic or abdominal surgery, present increased levels of pro-inflammatory cytokines [6]. IL-6 levels have been described as associated with post-operative complications, with an odds-ratio of 56.4 for the highest levels in a cohort of 96 patients benefiting from thoracic surgery [7]. Moreover, surgical patients often present a modification of their leukocyte profile with a redistribution of lymphocytes from the vascular area to lymphatic tissues, responsible for lymphopenia. Patients undergoing cardiac surgery present immunoparalysis described as an alteration in the expression of monocytic surface protein HLA-DR and an inhibition of monocytic and dendritic functions associated with an increase in the length of stay in intensive care units [6]. HLA-DR is implicated in defenses against pathogens and the reduction
$\mathrm{L}_{\mathrm{B}}$, lymphocytes $\mathrm{B} ; \mathrm{L}_{\mathrm{NK}}$, lymphocytes natural killers; TLR, tool like receptor; $\mathrm{TNF} \alpha$, tumor necrosis factor $\alpha$

in its expression is associated with mortality in severe infections [8] (Fig. 1).

All these elements allow us to suppose that adding surgical stress to a Covid-19 patient, or conversely developing this infection in an operated patient, may be deleterious in patients undergoing major surgery, particularly after cardiac surgery. This can also be transposed to patients requiring carcinological surgery. Thus, a recently published study by a Chinese team suggests an increased susceptibility to develop a severe SARS-Cov-2 infection in patients with underlying neoplasia, probably due to the underlying immunodepression. Thus, patients operated on or treated in the month prior to infection had a severe form in $75 \%$ of cases [9]. In addition, in a cohort study of 154 patients undergoing surgery for colorectal cancer, Xia et al. [10] demonstrated that patients with an N/L ratio $>2.8$ had an increase in postoperative complications with a 2 -year Hazard-ratio for mortality of 5.36 [1.95-14.90]. Because SARS-Cov-2 infection also increases N/L ratio, it questions the legitimity to operate these patients in such setting.

In the context of this epidemic, we must evaluate the risk of delaying an intervention for major surgery for more than $6-8$ weeks by taking into account the characteristics of the patient and his disease. The theoretical risk of postoperative complications is high, but the evolution of the disease while waiting for the resolution of the epidemic raises questions. 


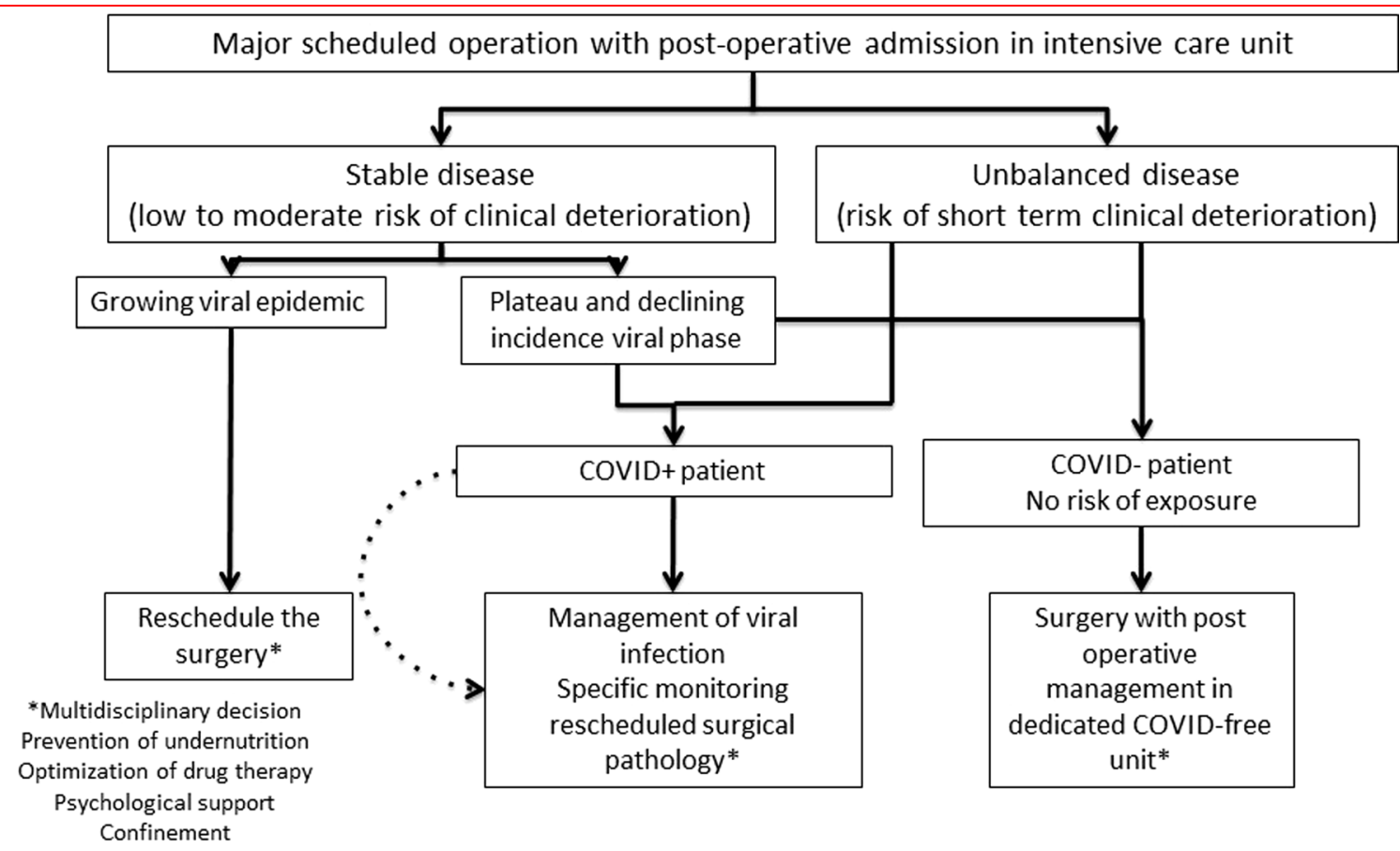

Fig. 2 Suggestion of a decisional algorithm for surgical scheduling in epidemic period of Covid-19

The possibilities of alternative therapies or monitoring must be considered on a case-by-case basis. Proposals have very recently been made by Tuech et al. [11] to best adapt the strategy according to the digestive surgical oncological situation, the co-morbidities of the patient and the risks of surgical complications. Restricted access to surgical operating theatres due to the epidemic will make it necessary to adapt the monitoring strategy and to optimize drug therapy to each patient. In the absence of alternative treatment, or waiting treatment, the patient will have to be informed of the appropriate strategy. In this context of expectation, care must be articulated around different key points as proposed by Tuech et al.:

(1) Discuss care in multidisciplinary meetings: personalized care plan best suited to the patient disease and the period of the epidemic (dematerialized meetings).

(2) Give preference to a therapeutic sequence that does not impose a strict surgical timing that cannot be achieved.

(3) Combating undernutrition and dehydration by promoting a balanced diet (nutritional supplement, hydration).

(4) Avoid significant damage to the immune system through aggressive treatments (case-by-case discussion of potential stand-by treatments).

(5) Avoid hospitalizations, visits, hospital stays that would favor contamination by the virus.
(6) Offer psychological support to patients who have to cope with the wait for major surgery (cardiac pathology, cancer) and with the management of the epidemic.

In Summary, SARS-Cov-2 infection as well as major surgery induce major inflammatory stress and deregulation of immunity, suggesting an increased risk of post-operative complications and mortality in this subgroup of patients. In the current pandemic context, the surgical indication of major scheduled must take into account the need for public health priorities in terms of the provision of intensive care units and dedicated personnel, as well as the aforementioned data on increased susceptibility to COVID-19 due to immunosuppressive changes around surgery. It would seem legitimate to decide on a case-by-case basis to operate only patients absolutely requiring surgery, based on a multidisciplinary discussion, making it possible to avoid an excess risk to the patient but also to spare the currently strained health system (Fig. 2).

\section{References}

1. Wu F, Zhao S, Yu B, Chen Y-M, Wang W, Song Z-G, Hu Y, Tao Z-W, Tian J-H, Pei Y-Y, Yuan M-L, Zhang Y-L, Dai F-H, Liu Y, Wang Q-M, Zheng J-J, Xu L, Holmes EC, Zhang Y-Z (2020) A new coronavirus associated with human respiratory disease in China. Nature 579:265-269 
2. Wu Z, McGoogan JM (2020) Characteristics of and important lessons from the coronavirus disease 2019 (COVID-19) outbreak in China: summary of a report of 72314 cases from the Chinese center for disease control and prevention. JAMA. https://doi.org/ 10.1001/jama.2020.2648

3. Xu Z, Shi L, Wang Y, Zhang J, Huang L, Zhang C, Liu S, Zhao P, Liu H, Zhu L, Tai Y, Bai C, Gao T, Song J, Xia P, Dong J, Zhao J, Wang F-S (2020) Pathological findings of COVID-19 associated with acute respiratory distress syndrome. Lancet Respir Med. https://doi.org/10.1016/S2213-2600(20)30076-X

4. Qin C, Zhou L, Hu Z, Zhang S, Yang S, Tao Y, Xie C, Ma K, Shang K, Wang W, Tian D-S (2020) Dysregulation of immune response in patients with COVID-19 in Wuhan, China. Clin Infect Dis. https://doi.org/10.1093/cid/ciaa248

5. Frink M, van Griensven M, Kobbe P, Brin T, Zeckey C, Vaske B, Krettek C, Hildebrand F (2009) IL-6 predicts organ dysfunction and mortality in patients with multiple injuries. Scand J Trauma Resusc Emerg Med 17:49

6. Corral-Velez V, Lopez-Delgado JC, Betancur-Zambrano NL, Lopez-Suñe N, Rojas-Lora M, Torrado H, Ballus J (2015) The inflammatory response in cardiac surgery: an overview of the pathophysiology and clinical implications. Inflamm Allergy Drug Targets 13:367-370

7. Kaufmann KB, Heinrich S, Staehle HF, Bogatyreva L, Buerkle H, Goebel U (2018) Perioperative cytokine profile during lung surgery predicts patients at risk for postoperative complicationsa prospective, clinical study. PLoS ONE 13:e0199807

8. Monneret G, Lepape A, Voirin N, Bohé J, Venet F, Debard A-L, Thizy H, Bienvenu J, Gueyffier F, Vanhems P (2006) Persisting low monocyte human leukocyte antigen-DR expression predicts mortality in septic shock. Intensive Care Med 32:1175-1183

9. Liang W, Guan W, Chen R, Wang W, Li J, Xu K, Li C, Ai Q, Lu W, Liang H, Li S, He J (2020) Cancer patients in SARS-CoV-2 infection: a nationwide analysis in China. Lancet Oncol 21:335-337

10. Xia L-J, Li W, Zhai J-C, Yan C-W, Chen J-B, Yang H (2020) Significance of neutrophil-to-lymphocyte ratio, platelet-to-lymphocyte ratio, lymphocyte-to-monocyte ratio and prognostic nutritional index for predicting clinical outcomes in T1-2 rectal cancer. BMC Cancer 20:208

11. Tuech JJ, Gangloff A, Di Fiore F, Brigand C, Michel P, Pocard M, Schwarz L (2020) Strategy for the Practice of Digestive surgery and Surgerical Oncology in COVID-19 Outbreak Situation. J Visc Surg. https://doi.org/10.1016/j.jchirv.2020.03.007

Publisher's Note Springer Nature remains neutral with regard to jurisdictional claims in published maps and institutional affiliations. 